\title{
Probiotics are effective at preventing Clostridium difficile-associated diarrhea: a systematic review and meta-analysis
}

This article was published in the following Dove Press journal:

International Journal of General Medicine

22 February 2016

Number of times this article has been viewed

\author{
Christine SM Lau', \\ Ronald S Chamberlain ${ }^{1-3}$ \\ 'Department of Surgery, Saint \\ Barnabas Medical Center, Livingston, \\ NJ, USA; ${ }^{2}$ Saint George's University \\ School of Medicine, Grenada, West \\ Indies; ${ }^{3}$ Department of Surgery, \\ New Jersey Medical School, Rutgers \\ University, Newark, NJ, USA
}

Introduction: Clostridium difficile infection (CDI) is the leading cause of antibiotic-associated diarrhea. CDI has increased in incidence and severity over the past decade, and is a growing worldwide health problem associated with substantial health care costs and significant morbidity and mortality. This meta-analysis examines the impact of probiotics on the incidence of Clostridium difficile-associated diarrhea (CDAD) among children and adults, in both hospital and outpatient settings.

Methods: A comprehensive literature search of all published randomized control trials (RCTs) assessing the use of probiotics in the prevention of CDAD in patients receiving antibiotic therapy was conducted, and the incidence of CDAD was analyzed.

Results: Twenty-six RCTs involving 7,957 patients were analyzed. Probiotic use significantly reduced the risk of developing CDAD by $60.5 \%$ (relative risk $[R R]=0.395 ; 95 \%$ confidence interval $[\mathrm{CI}], 0.294-0.531 ; P<0.001)$. Probiotics proved beneficial in both adults and children (59.5\% and $65.9 \%$ reduction), especially among hospitalized patients. Lactobacillus, Saccharomyces, and a mixture of probiotics were all beneficial in reducing the risk of developing CDAD (63.7\%, 58.5\%, and 58.2\% reduction).

Conclusion: Probiotic supplementation is associated with a significant reduction in the risk of developing CDAD in patients receiving antibiotics. Additional studies are required to determine the optimal dose and strain of probiotic.

Keywords: probiotics, Clostridium difficile-associated diarrhea, antibiotic-associated diarrhea

\section{Introduction}

Clostridium difficile, a Gram-positive, spore forming, and toxin-producing anaerobic rod bacterium, is the leading cause of hospital- and community-acquired antibioticassociated diarrhea (AAD) in the Western world. ${ }^{1,2}$ C. difficile infection (CDI) is a growing worldwide health problem associated with substantial health care costs and significant morbidity and mortality. ${ }^{2}$ The incidence of CDI in the United States is rapidly increasing, and is estimated to affect approximately $1 \%$ of all hospitalized patients, increasing length of stay by $55 \%$, and US health care system costs by USD1-3 billion annually. . $^{1,3,4}$

C. difficile-associated diarrhea (CDAD) occurs most often in elderly and hospitalized patients receiving broad-spectrum antibiotics. ${ }^{2,5-8}$ Antibiotic exposure is considered the most significant risk factor for CDI, and several drugs have been implicated in high CDAD rates including cephalosporins, fluoroquinolones, penicillins, and clindamycin. ${ }^{1,9-11}$ Other CDAD risk factors include the use of proton-pump inhibitors, $\mathrm{H}_{2}$ antagonists, methotrexate,
Correspondence: Ronald S Chamberlain Department of Surgery, Saint Barnabas Medical Center, 94 Old Short Hills Road, Livingston, NJ 07039, USA

Tel +19733225195

Fax + I 973322 247|

Email rchamberlain@barnabashealth.org
International Journal of General Medicine 2016:9 27-37

(c) (1) (8) ( ) 2016 Lau and Chamberlan. This work is published and licensed by Dove Medical Press Limited. The full terms of this license are avalable at https://wwr.dovepress. (c) the work you herbby accept the Tems. Non-commercial uses of the work are permitted without any further permision from Dove Medical Press Limited, provided the work is properly atributed. For permission for commercial use of this work, please see paragraphs 4.2 and 5 of our Terms (https://www.dovepress. com/terms.php).
Dovepress

http://dx.doi.org/10.2147/IJGM.S98280

27 
and the presence of existing gastrointestinal pathologies, such as inflammatory bowel disease. ${ }^{1,9}$

Most approaches for the prevention of CDI to date have focused on limiting the spread of CDI. The most common methods are early detection and isolation, contact precautions, and appropriate hand hygiene. A number of studies have focused on the role of environmental cleaning to eradicate CDI in the health care environment, including the use of environment disinfectants as well as chlorhexidine patient baths, but have shown limited success. ${ }^{12,13}$

More recently, probiotics have been proposed for the prevention and treatment of a variety of gastrointestinal conditions, including diarrhea. Normal intestinal flora is an important barrier against pathogenic bacteria, and disruption of this normal flora with antibiotic use can lead to diarrhea. ${ }^{14,15}$ Probiotics are live microbial food supplements and have been hypothesized to counteract disturbances in intestinal flora and reduce colonization by pathogenic bacteria. ${ }^{14,15}$ Various species of probiotics have been studied, with the most common being within the Lactobacillus and Bifidobacterium genus. More recently, Saccharomyces boulardii, a yeast, has also been considered a probiotic. ${ }^{15}$

Hempel et $\mathrm{al}^{16}$ reported a $42 \%$ reduction in the risk of developing AAD with the use of probiotics (relative risk $[\mathrm{RR}]=0.58 ; 95 \%$ confidence interval [CI], 0.50-0.68; $P<0.001$ ). In a meta-analysis by Johnston et al, ${ }^{17}$ a $66 \%$ reduction in the risk of $\mathrm{CDAD}$ with the use of probiotics (RR $=0.34 ; 95 \%$ CI, 0.24-0.49; $P<0.001$ ) was observed. A Cochrane Review reported similar results with a $64 \%$ reduction in the risk of CDAD. ${ }^{18}$

A significant number of more contemporary randomized controlled trials (RCTs) not included in the Johnston et al study ${ }^{17}$ or the Cochrane Review have recently been published, which address probiotic use, and have yielded conflicting results. ${ }^{19-22}$ Shan et $\mathrm{al}^{20}$ studied probiotic supplementation in 283 children between the ages of 6 months and 14 years (139 receiving $S$. boulardii and 144 in the control group), and reported one case of CDAD in the probiotic group and eight cases in the control group $(\mathrm{RR}=0.13 ; 95 \% \mathrm{CI}, 0.02-1.05$, $P<0.05)$. Conversely, Allen et $\mathrm{al}^{19}$ published results from the largest RCT, involving 2,941 hospitalized patients $>65$ years (1,470 patients receiving probiotics and 1,471 patients in the control group) across five hospitals in the United Kingdom, and reported no significant reduction in CDAD incidence with the use of a multistrain preparation of Lactobacillus and Bifidobacterium $(\mathrm{RR}=0.71 ; 95 \% \mathrm{CI}, 0.34-1.47 ; P=0.35)$.

Given the high morbidity and mortality associated with $\mathrm{CDI}$, and conflicting results among published RCTs, this meta-analysis provides an updated analysis on the efficacy of probiotics in the prevention of CDAD, for both adults and children, in the hospital and outpatient settings.

\section{Methods \\ Study selection}

A comprehensive search of all published RCTs evaluating probiotics to prevent CDAD was conducted using PubMed, Cochrane Central Registry of Controlled Trials, and Google Scholar (1966-2015). Additional citations were searched, using the references of the articles retrieved from prior publications. The last search was conducted on October 10, 2015, and only articles written in English were considered. Keywords used in the search included combinations of "probiotics", "Lactobacillus", "Bifidobacterium", "Saccharomyces", "Clostridium difficile", "Clostridium difficile-associated diarrhea", "antibiotic associated diarrhea", and "diarrhea". The following inclusion criteria were used: RCTs comparing the use of any strain or dose of a specified probiotic with a placebo or "no intervention" control group, probiotics initiated within 3 days of starting antibiotics and continued for at least the entire duration of antibiotic treatment. In case of duplicate publications, only the most recent and updated report of the clinical trial was included.

\section{Data extraction}

Articles retrieved from the searches were assessed for eligibility, and data pertaining to patients, intervention, control groups, outcomes, and methodology were abstracted (Figure 1). The clinical outcome of interest was incidence of CDAD (diarrhea and positive stool cytotoxin assay or culture), among children and adults, in both the hospital and outpatient setting.

\section{Statistical analysis}

For each trial, RR with a 95\% CI for CDAD was calculated. Meta-analysis of the pooled data was performed using the Comprehensive Meta-Analysis software Version 3 (Biostat, Englewood, NJ, USA). For studies reporting zero events in any group, a continuity correction factor of 0.5 was adopted to calculate the RR and variance. If there was a zero event in both groups, the RR was not calculable and the study was excluded from the meta-analysis. Both the fixed-effects model and random-effects model were considered, depending on the heterogeneity of the included studies. To assess the heterogeneity between studies, both Cochrane's $Q$ statistic and $I^{2}$ statistic were used. Heterogeneity was considered statistically significant when $P<0.05$ or $P^{2}>50$. If heterogeneity 


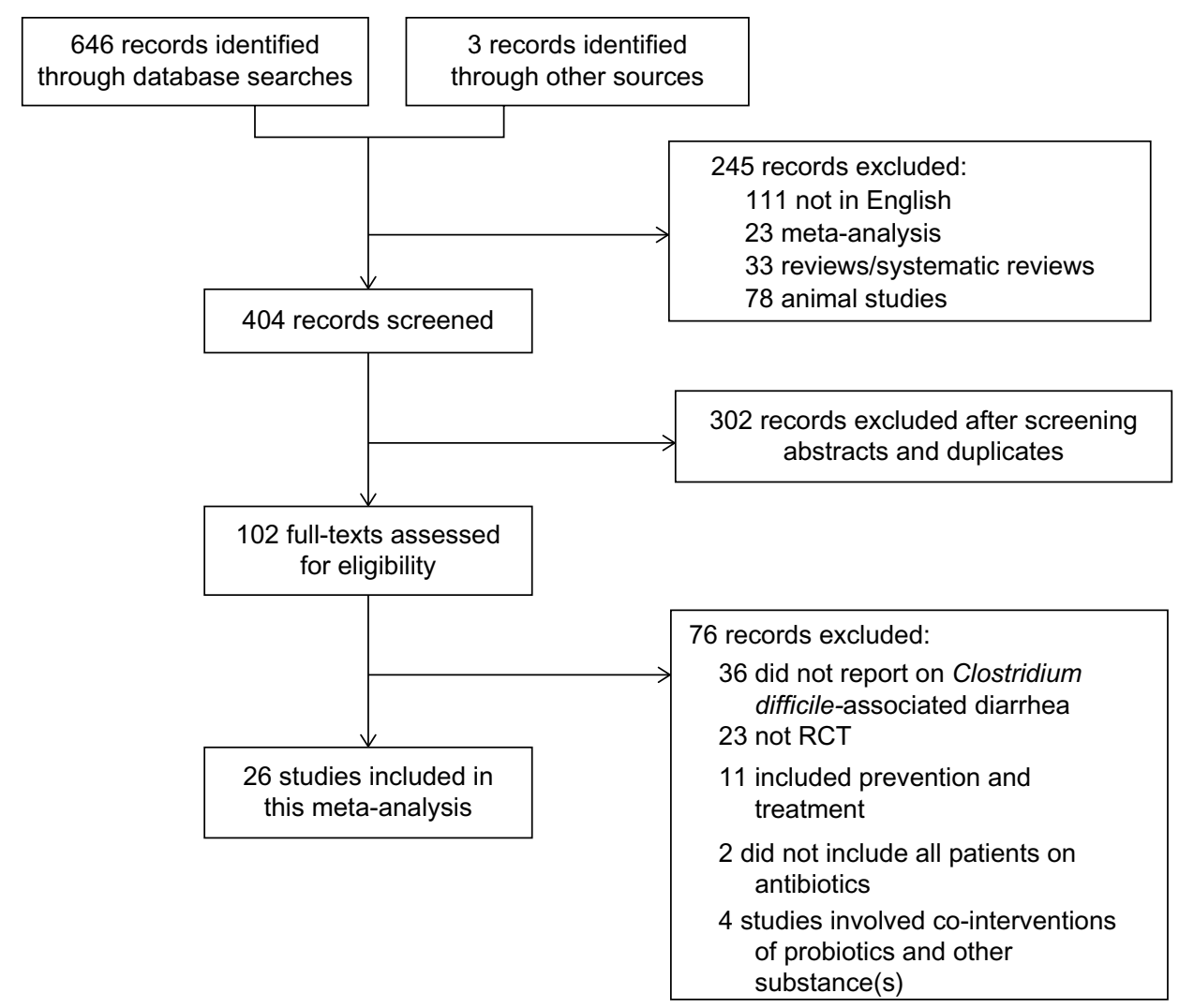

Figure I CONSORT diagram of the study selection process.

Abbreviations: RCT, randomized control trial; CONSORT, Consolidated Standards of Reporting Trials.

was observed, data were analyzed using a random-effects model. Conversely, in the absence of heterogeneity, a fixedeffects model was assumed. The publication bias regarding the RR of CDAD in patients receiving probiotics was first visually evaluated by a funnel plot, and further evaluated using Egger's and Begg's tests. A two-tailed $P$-value of $<0.05$ was considered statistically significant. Subgroup analysis was performed based on the genus of probiotics (Lactobacillus, Bifidobacterium, Saccharomyces, and a mixture of probiotics), the age of the patient (pediatric patients $<18$ years and adults), and the health care setting (hospitalized inpatient or outpatient).

\section{Results}

\section{Demographic characteristics of the studies}

A total of 26 RCTs evaluating the use of probiotics in the prevention of CDAD were identified, involving a total of 7,957 patients (Table 1). Approximately 4,124 patients received probiotic supplementation, and 3,833 patients received either placebo or "no treatment". Among patients who received probiotics, 2,273 received Lactobacillus, 1,757 received Saccharomyces, and 3,927 received a mixture of probiotics.
None of the studies compared Bifidobacterium alone with a placebo group.

\section{Effects of probiotics on CDAD}

Data on the incidence of CDAD (defined as diarrhea and positive stool cytotoxin assay or culture) in both the probiotic group and the placebo group were reported in all trials. Fewer patients in the probiotics group developed CDAD, compared to the control group who received placebo or no supplement $(62 / 4,124$ [1.5\%] versus $145 / 3,833$ [3.8\%]). There was no significant heterogeneity between trials $\left(P=0.751, I^{2}=0.000\right)$, and a fixed-effects model was assumed. Meta-analysis showed a significantly lower risk of developing CDAD in the probiotics group compared to the control group $(\mathrm{RR}=0.395$; 95\% CI, 0.294-0.531; $P<0.001$; Figure 2).

Subgroup analysis identified that Lactobacillus, Saccharomyces, or a mixture of probiotics were beneficial in reducing the risk of developing CDAD $(\mathrm{RR}=0.363 ; 95 \%$ CI, 0.225-0.585; $P<0.001$ for Lactobacillus; RR =0.415; 95\% CI, 0.217-0.796; $P=0.008$ for Saccharomyces; and $\mathrm{RR}=0.418 ; 95 \% \mathrm{CI}, 0.263-0.664 ; P<0.001$ for mixture of probiotics; Figure 3). Probiotics were beneficial for both adults $(\mathrm{RR}=0.405 ; 95 \% \mathrm{CI}, 0.294-0.556 ; P<0.001)$ 


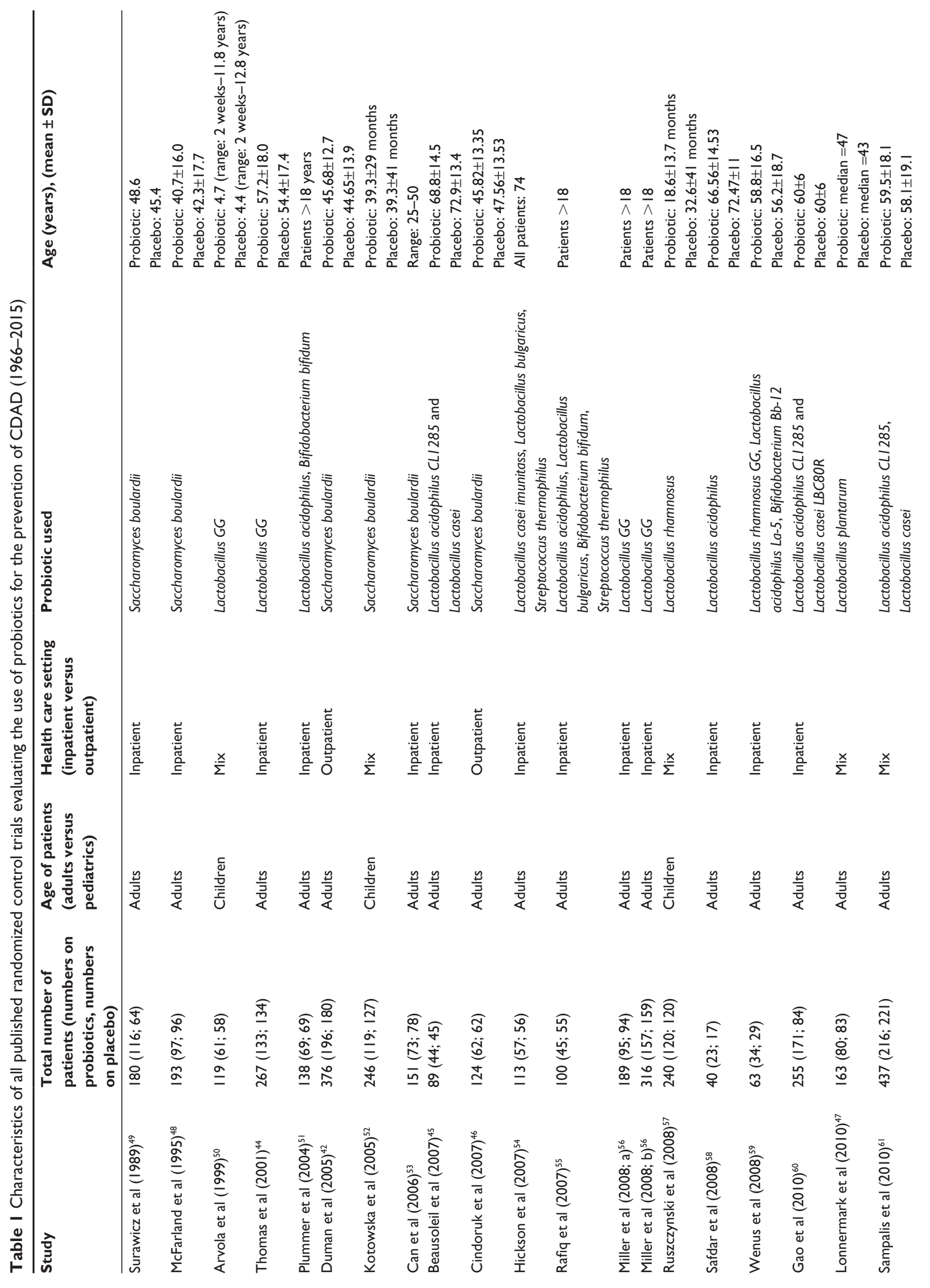



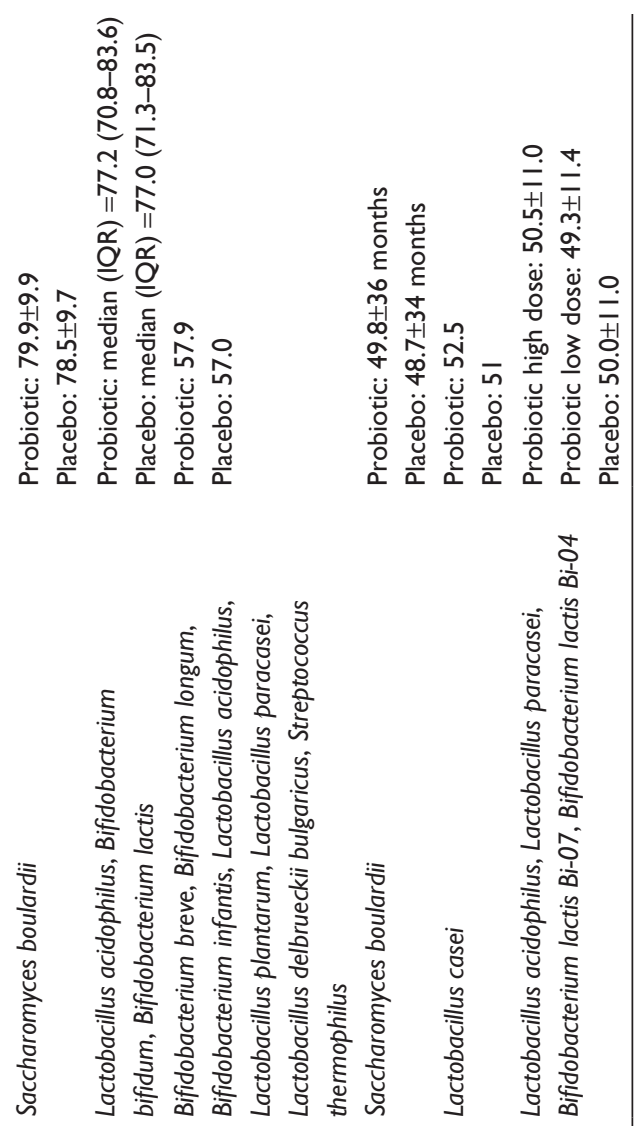

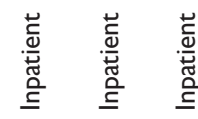

$\begin{array}{lll}\frac{n}{5} & \frac{n}{5} & \frac{n}{5} \\ \frac{0}{2} & \frac{7}{4}\end{array}$

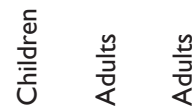

$\frac{\breve{s}}{\frac{3}{4}}$

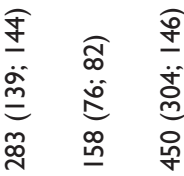

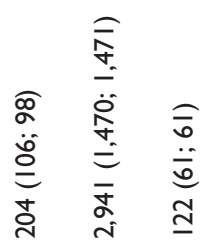

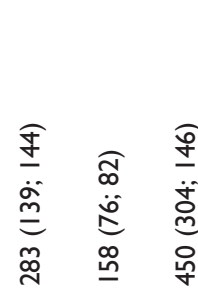

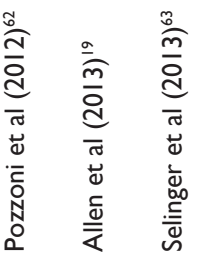

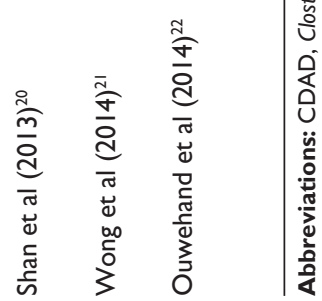

and children $(\mathrm{RR}=0.341 ; 95 \% \mathrm{CI}, 0.153-0.759 ; P=0.008$; Figure 4). Hospitalized patients were more likely to benefit from probiotic use compared to outpatients $(\mathrm{RR}=0.390$; 95\% CI, $0.283-0.538 ; P<0.001$ versus $\mathrm{RR}=0.306 ; 95 \% \mathrm{CI}$, $0.013-7.470 ; P=0.468$; Figure 5).

\section{Subgroup analysis based on type of C. difficile testing}

Most studies utilized stool culture and cytotoxin testing, or enzyme immunoassay (EIA) in diagnosing $C$. difficile. One study used EIA but stated that polymerase chain reaction was available if required. A few studies were nonspecific and either did not state the technique utilized or simply stated that C. difficile was diagnosed according to hospital protocol.

Subgroup analysis based on the testing method (stool culture and cytotoxin versus EIA) identified that probiotics were beneficial in reducing the risk of CDAD with both the stool cytotoxin $(\mathrm{RR}=0.271 ; 95 \% \mathrm{CI}, 0.131-0.561 ; P<0.001)$ and EIA testing methods ( $\mathrm{RR}=0.431 ; 95 \% \mathrm{CI}, 0.288-0.647$; $P<0.001)$. There was no significant difference between the benefit derived from either testing method $(P=0.536)$.

\section{Publication bias}

A funnel plot was used to qualitatively assess for publication bias, and Egger's and Begg's tests were conducted to calculate publication bias. There was no obvious evidence of asymmetry on the funnel plot (Figure 6). Furthermore, there was no evidence of publication bias for the primary end point of this study (RR of CDAD in patients receiving probiotics) by either the Egger's $(P=0.748)$ or Begg's test $(P=0.747)$.

\section{Discussion}

C. difficile was first described by Hall and $\mathrm{O}^{\prime} \mathrm{Tool}^{23}$ as part of the intestinal microflora in neonates, and represents the leading cause of AAD. ${ }^{24,25}$ Prior to the year 2000, the rate of CDI in the USA did not vary greatly from year to year and has been relatively stable at $30-40$ cases per 100,000 population. ${ }^{26}$ In 2001, a sudden spike in CDAD rates occurred, rising to approximately 50 cases per 100,000 population, and this incidence has continued to rise by approximately $25 \%$ each year. ${ }^{26}$ In a retrospective analysis of the US National Hospital Discharge Survey between 2001 and 2010, Reveles et al ${ }^{27}$ reported that the incidence of CDI among hospitalized patients nearly doubled from 4.5 CDI discharges per 1,000 total adult discharges in 2001 to $8.2 \mathrm{CDI}$ discharges per 1,000 adult discharges in 2010. Overall mortality also increased from $6.6 \%$ in 2001 to $7.2 \%$ in $2010 .{ }^{27}$ 
Study

\begin{tabular}{|c|c|c|c|c|}
\hline & $\begin{array}{l}\text { Risk } \\
\text { ratio }\end{array}$ & $\begin{array}{l}\text { Lower } \\
\text { limit }\end{array}$ & $\begin{array}{l}\text { Upper } \\
\text { limit }\end{array}$ & $P$-value \\
\hline Surawicz et al $(1989)^{49}$ & 0.331 & 0.082 & 1.340 & \\
\hline McFarland et al $(1995)^{48}$ & 0.742 & 0.171 & 3.229 & \\
\hline Arvola et al (1999) $)^{50}$ & 0.951 & 0.061 & 14.850 & \\
\hline Thomas et al $(2001)^{44}$ & 0.672 & 0.114 & 3.955 & \\
\hline Plummer et al $(2004)^{51}$ & 0.400 & 0.080 & 1.992 & \\
\hline Duman et al $(2005)^{42}$ & 0.306 & 0.013 & 7.470 & \\
\hline Kotowska et al $(2005)^{52}$ & 0.320 & 0.090 & 1.135 & \\
\hline Can et al $(2006)^{53}$ & 0.214 & 0.010 & 4.374 & \\
\hline Beausoleil et al $(2007)^{45}$ & 0.146 & 0.019 & 1.139 & \\
\hline Hickson et al $(2007)^{54}$ & 0.052 & 0.003 & 0.868 & \\
\hline Rafiq et al $(2007)^{55}$ & 0.278 & 0.114 & 0.675 & \\
\hline Miller et al $(2008 ; a)^{56}$ & 0.565 & 0.171 & 1.868 & \\
\hline Miller et al $(2008 ; b)^{56}$ & 5.063 & 0.245 & 104.628 & \\
\hline Ruszczynski et al $(2008)^{57}$ & 0.429 & 0.114 & 1.618 & \\
\hline Safdar et al $(2008)^{58}$ & 0.250 & 0.011 & 5.786 & \\
\hline Wenus et al $(2008)^{59}$ & 0.286 & 0.012 & 6.756 & \\
\hline Gao et al $(2010)^{60}$ & 0.221 & 0.105 & 0.464 & \\
\hline Lonnermark et al $(2010)^{47}$ & 3.111 & 0.129 & 75.264 & \\
\hline Sampalis et al $(2010)^{61}$ & 0.256 & 0.029 & 2.270 & \\
\hline Pozzoni et al (2012) ${ }^{62}$ & 1.387 & 0.237 & 8.125 & \\
\hline Allen et al $(2013)^{19}$ & 0.706 & 0.339 & 1.474 & \\
\hline Shan et al $(2013)^{20}$ & 0.129 & 0.016 & 1.022 & \\
\hline Wong et al $(2013)^{21}$ & 0.359 & 0.015 & 8.688 & \\
\hline Ouwehand et al (2014) & 0.360 & 0.127 & 1.019 & \\
\hline Overall & 0.395 & 0.294 & 0.531 & \\
\hline
\end{tabular}

Risk ratio and $95 \% \mathrm{Cl}$

Relative

weight

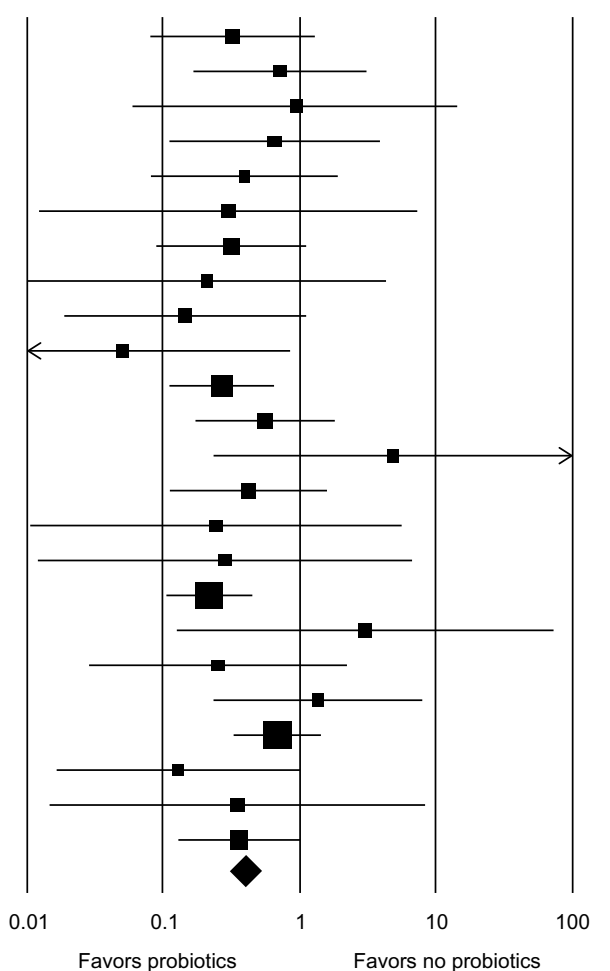

4.48

4.06

1.16

2.79

3.40

0.86

5.47

0.96

2.08

1.10

11.13

6.14

0.96

4.97

0.89

0.88

15.92

0.86

1.84

2.81

16.21

2.05

0.86

8.11

Figure 2 Forest plot evaluating the RR of CDAD associated with probiotic use.

Abbreviations: $\mathrm{RR}$, relative risk; $\mathrm{Cl}$, confidence interval; $\mathrm{CDAD}$, Clostridium difficile-associated diarrhea.

Group by
probiotic
Lactobacillus
Lactobacillus
Lactobacillus
Lactobacillus
Lactobacillus
Lactobacillus
Lactobacillus
Lactobacillus
Lactobacillus
Lactobacillus
Lactobacillus
Lactobacillus
Mix
Mix
Mix
Mix
Mix
Mix
Mix
Saccharomyces
Saccharomyces
Saccharomyces
Saccharomyces
Saccharomyces
Saccharomyces
Saccharomyces
Saccharomyces
Overall

Study

Arvola et al (1999) $)^{50}$ Thomas et al $(2001)^{44}$ Beausoleil et al $(2007)^{45}$ Miller et al $(2008 ; a)^{56}$ Miller et al $(2008 ; b)^{56}$ Ruszczynski et al $(2008)^{57}$ Safdar et al $(2008)^{58}$ Gao et al $(2010)^{60}$ Lonnermark et al (2010) $)^{47}$ Sampalis et al $(2010)^{61}$ Wong et al $(2014)^{21}$

Plummer et al (2004) $)^{51}$ Hickson et al (2007) ${ }^{54}$ Rafiq et al $(2007)^{55}$

Wenus et al (2008) $)^{59}$ Allen et al (2013) ${ }^{19}$ Ouwehand et al $(2014)^{22}$

Surawicz et al (1989) ${ }^{49}$ McFarland et al $(1995)^{48}$ Duman et al $(2005)^{42}$ Kotowska et al $(2005)^{52}$ Can et al $(2006)^{53}$ Pozzoni et al (2012) ${ }^{62}$ Shan et al $(2013)^{20}$
Statistics for each study Risk Lower Upper

ratio limit limit $\boldsymbol{P}$-value

$\begin{array}{llll}0.951 & 0.061 & 14.850 & 0.971\end{array}$

$\begin{array}{llll}0.672 & 0.114 & 3.955 & 0.660\end{array}$

$\begin{array}{llll}0.146 & 0.019 & 1.139 & 0.066\end{array}$

$\begin{array}{llll}0.565 & 0.171 & 1.868 & 0.350\end{array}$

$\begin{array}{llll}5.063 & 0.245 & 104.628 & 0.294\end{array}$

$\begin{array}{llrr}0.429 & 0.114 & 1.618 & 0.211\end{array}$

$\begin{array}{llll}0.250 & 0.011 & 5.786 & 0.387\end{array}$

$\begin{array}{llll}0.221 & 0.105 & 0.484 & 0.000\end{array}$

$\begin{array}{llll}3.111 & 0.129 & 75.264 & 0.485\end{array}$

$\begin{array}{llll}0.256 & 0.029 & 2.270 & 0.221\end{array}$

$\begin{array}{llll}0.359 & 0.015 & 8.688 & 0.529\end{array}$

$\begin{array}{llll}0.363 & 0.225 & 0.585 & 0.000\end{array}$

$\begin{array}{llll}0.400 & 0.080 & 1.992 & 0.263\end{array}$

$\begin{array}{llll}0.052 & 0.003 & 0.868 & 0.040\end{array}$

$\begin{array}{llll}0.278 & 0.114 & 0.675 & 0.005\end{array}$

$\begin{array}{llll}0.286 & 0.012 & 6.756 & 0.438\end{array}$

$\begin{array}{llll}0.706 & 0.339 & 1.474 & 0.354\end{array}$

$\begin{array}{llll}0.360 & 0.127 & 1.019 & 0.054\end{array}$

$\begin{array}{llll}0.418 & 0.263 & 0.664 & 0.000\end{array}$

$\begin{array}{llll}0.331 & 0.082 & 1.340 & 0.121\end{array}$

$\begin{array}{llll}0.742 & 0.171 & 3.229 & 0.691\end{array}$

$\begin{array}{llll}0.306 & 0.013 & 7.470 & 0.468\end{array}$

$\begin{array}{llll}0.320 & 0.090 & 1.135 & 0.078\end{array}$

$\begin{array}{llll}0.214 & 0.010 & 4.374 & 0.316\end{array}$

$\begin{array}{llll}1.387 & 0.237 & 8.125 & 0.717\end{array}$

$\begin{array}{llll}0.129 & 0.016 & 1.022 & 0.052\end{array}$

$\begin{array}{llll}0.415 & 0.217 & 0.796 & 0.008\end{array}$

$\begin{array}{llll}0.395 & 0.294 & 0.531 & 0.000\end{array}$

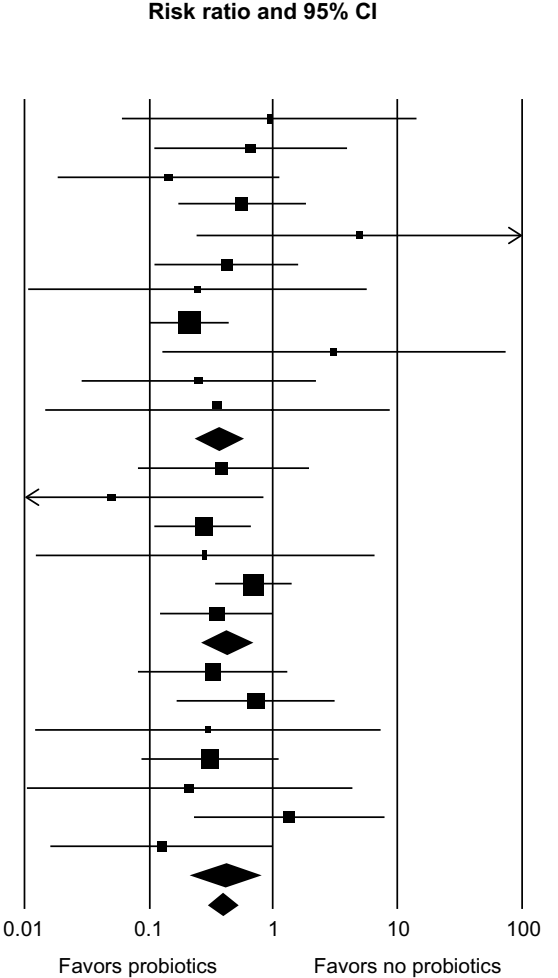

Relative weight

3.02

7.25

5.40
15.96

2.49

12.91

2.31

41.39

2.25
4.78

2.25

8.33

2.70

27.26

2.15

39.70

19.86

21.67

19.60

4.15

26.45

4.65

13.58

9.93

Figure 3 Forest plot evaluating the RR of CDAD associated with various species of probiotic use.

Abbreviations: $\mathrm{RR}$, relative risk; $\mathrm{Cl}$, confidence interval; $\mathrm{CDAD}$, Clostridium difficile-associated diarrhea. 
Group by population

Study

Adults

Adults

Adults

Adults

Adults

Adults

Adults

Adults

Adults

Adults

Adults

Adults

Adults

Adults

Adults

Adults

Adults

Adults

Adults

Adults

Adults

Children

Children

Children

Children

Children

Overall
Statistics for each study

Risk Lower Upper

ratio limit limit $P$-value

$\begin{array}{llll}0.331 & 0.082 & 1.340 & 0.121\end{array}$

$\begin{array}{llll}0.742 & 0.171 & 3.229 & 0.691\end{array}$

$\begin{array}{llll}0.672 & 0.114 & 3.955 & 0.660\end{array}$

$\begin{array}{llll}0.400 & 0.080 & 1.992 & 0.263\end{array}$

$\begin{array}{llll}0.306 & 0.013 & 7.470 & 0.468\end{array}$

$\begin{array}{llll}0.214 & 0.010 & 4.374 & 0.316\end{array}$

$\begin{array}{llll}0.146 & 0.019 & 1.139 & 0.066\end{array}$

$\begin{array}{llll}0.052 & 0.003 & 0.868 & 0.040\end{array}$

$\begin{array}{llll}0.278 & 0.114 & 0.675 & 0.005\end{array}$

$\begin{array}{llll}0.565 & 0.171 & 1.868 & 0.350\end{array}$

$\begin{array}{llll}5.063 & 0.245 & 104.628 & 0.294\end{array}$

$0.250 \quad 0.011$

$\begin{array}{llll}0.286 & 0.012 & 6.756 & 0.438\end{array}$

$\begin{array}{llll}0.221 & 0.105 & 0.464 & 0.000\end{array}$

$\begin{array}{llll}3.111 & 0.129 & 75.264 & 0.485\end{array}$

$\begin{array}{llll}0.256 & 0.029 & 2.270 & 0.221\end{array}$

$\begin{array}{llll}1.387 & 0.237 & 8.125 & 0.717\end{array}$

$\begin{array}{llll}0.706 & 0.339 & 1.474 & 0.354\end{array}$

$\begin{array}{llll}0.359 & 0.015 & 8.688 & 0.529\end{array}$

$\begin{array}{llll}0.360 & 0.127 & 1.019 & 0.054\end{array}$

$\begin{array}{llll}0.405 & 0.294 & 0.556 & 0.000\end{array}$

$\begin{array}{llll}0.951 & 0.061 & 14.850 & 0.971\end{array}$

$\begin{array}{llll}0.320 & 0.090 & 1.135 & 0.078\end{array}$

$\begin{array}{llll}0.429 & 0.114 & 1.618 & 0.211\end{array}$

$\begin{array}{llll}0.129 & 0.016 & 1.022 & 0.052\end{array}$

$0.341 \quad 0.153$

$0.395 \quad 0.294$

0.759

0.008

0.000
Risk ratio and $95 \% \mathrm{Cl}$

Relative

weight

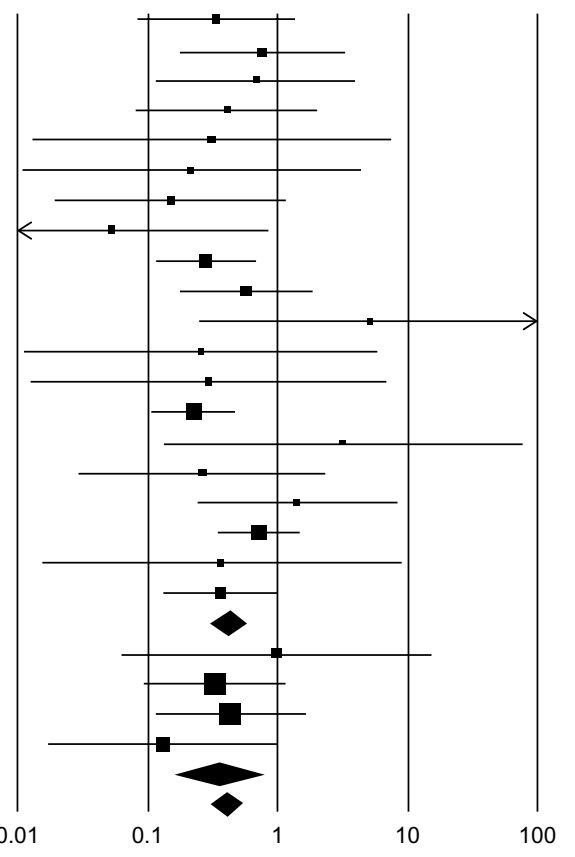

Favors probiotics
5.19

4.70

3.23

3.94

1.00

1.11

2.41

1.28

12.89

7.11

1.11

1.03

1.01

18.44

1.00

2.13

3.25

18.78

1.00

9.39

8.50

40.08

36.37

15.05

Figure 4 Forest plot evaluating the RR of CDAD associated with probiotic use in adult and pediatric populations.

Abbreviations: $\mathrm{RR}$, relative risk; $\mathrm{Cl}$, confidence interval; $\mathrm{CDAD}$, Clostridium difficile-associated diarrhea.

\begin{tabular}{|c|c|c|c|c|c|}
\hline \multirow{2}{*}{$\begin{array}{l}\text { Group by } \\
\text { setting }\end{array}$} & \multirow[t]{2}{*}{ Study } & \multicolumn{4}{|c|}{ Statistics for each study } \\
\hline & & $\begin{array}{l}\text { Risk } \\
\text { ratio }\end{array}$ & $\begin{array}{l}\text { Lower } \\
\text { limit }\end{array}$ & $\begin{array}{l}\text { Upper } \\
\text { limit }\end{array}$ & $P$-value \\
\hline Inpatient & Surawicz et al $(1989)^{49}$ & 0.331 & 0.082 & 1.340 & 0.121 \\
\hline Inpatient & McFarland et al $(1995)^{48}$ & 0.742 & 0.171 & 3.229 & 0.691 \\
\hline Inpatient & Thomas et al $(2001)^{44}$ & 0.672 & 0.114 & 3.955 & 0.660 \\
\hline Inpatient & Plummer et al $(2004)^{51}$ & 0.400 & 0.080 & 1.992 & 0.263 \\
\hline Inpatient & Can et al $(2006)^{53}$ & 0.214 & 0.010 & 4.374 & 0.316 \\
\hline Inpatient & Beausoleil et al $(2007)^{45}$ & 0.146 & 0.019 & 1.139 & 0.066 \\
\hline Inpatient & Hickson et al $(2007)^{54}$ & 0.052 & 0.003 & 0.868 & 0.040 \\
\hline Inpatient & Rafiq et al $(2007)^{55}$ & 0.278 & 0.114 & 0.675 & 0.005 \\
\hline Inpatient & Miller et al $(2008 ; a)^{56}$ & 0.565 & 0.171 & 1.868 & 0.350 \\
\hline Inpatient & Miller et al $(2008 ; b)^{56}$ & 5.063 & 0.245 & 104.628 & 0.294 \\
\hline Inpatient & Safdar et al $(2008)^{58}$ & 0.250 & 0.011 & 5.786 & 0.387 \\
\hline Inpatient & Wenus et al $(2008)^{59}$ & 0.286 & 0.012 & 6.756 & 0.438 \\
\hline Inpatient & Gao et al $(2010)^{60}$ & 0.221 & 0.105 & 0.464 & 0.000 \\
\hline Inpatient & Pozzoni et al $(2012)^{62}$ & 1.387 & 0.237 & 8.125 & 0.717 \\
\hline Inpatient & Allen et al $(2013)^{19}$ & 0.706 & 0.339 & 1.474 & 0.354 \\
\hline Inpatient & Shan et al $(2013)^{20}$ & 0.129 & 0.016 & 1.022 & 0.052 \\
\hline Inpatient & Wong et al $(2014)^{21}$ & 0.359 & 0.015 & 8.688 & 0.529 \\
\hline Inpatient & Ouwehand et al (2014) 22 & 0.360 & 0.127 & 1.019 & 0.054 \\
\hline Inpatient & & 0.390 & 0.283 & 0.538 & 0.000 \\
\hline Mix & Arvola et al $(1999)^{50}$ & 0.951 & 0.061 & 14.850 & 0.971 \\
\hline Mix & Kotowska et al $(2005)^{52}$ & 0.320 & 0.090 & 1.135 & 0.078 \\
\hline Mix & Ruszczynski et al $(2008)^{57}$ & 0.429 & 0.114 & 1.618 & 0.211 \\
\hline Mix & Lonnermark et al $(2010)^{47}$ & 3.111 & 0.129 & 75.264 & 0.485 \\
\hline Mix & Sampalis et al $(2010)^{61}$ & 0.256 & 0.029 & 2.270 & 0.221 \\
\hline Mix & & 0.431 & 0.197 & 0.944 & 0.035 \\
\hline Outpatient & Duman et al $(2005)^{42}$ & 0.306 & 0.013 & 7.470 & 0.468 \\
\hline Outpatient & & 0.306 & 0.013 & 7.470 & 0.468 \\
\hline Overall & & 0.395 & 0.294 & 0.531 & 0.000 \\
\hline
\end{tabular}

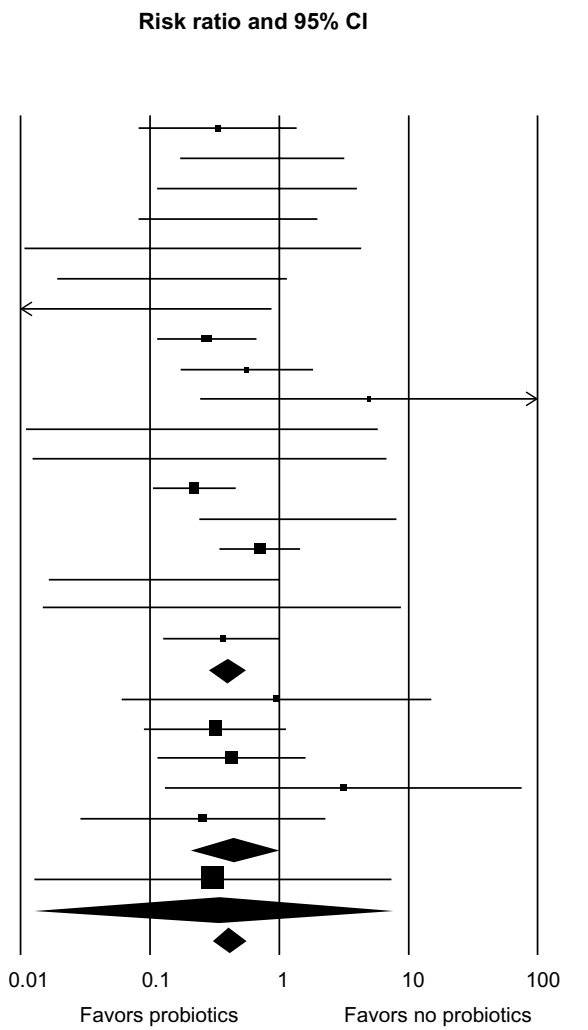

Relative

weight

5.29

4.78

3.29

4.01

1.13

2.45

1.30

13.12

7.24

1.13

1.05

1.03

18.77

3.31

19.11

2.42

1.02

9.56

8.11

38.26

34.73

6.04

12.86

100.00

Figure 5 Forest plot evaluating the RR of CDAD associated with probiotic use in both inpatient and outpatient populations.

Abbreviations: $\mathrm{RR}$, relative risk; $\mathrm{Cl}$, confidence interval; $\mathrm{CDAD}$, Clostridium difficile-associated diarrhea. 


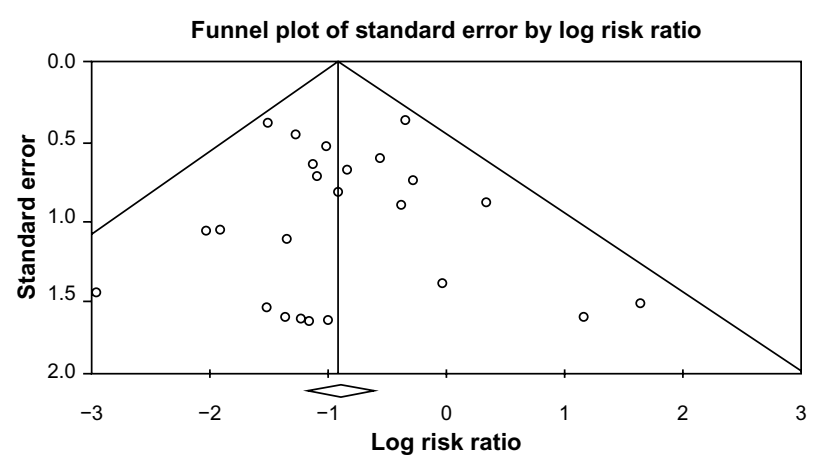

Figure 6 Funnel plot assessing publication bias (analyzing the effect of probiotic supplementation on the incidence of CDAD).

Abbreviation: CDAD, Clostridium difficile-associated diarrhea.

The rise in CDI has been attributed to various factors, including antibiotic resistance and the emergence of new C. difficile strains. The toxogenic $C$. difficile NAP1/BI/027 strain was discovered in 2002, and found to be associated with more severe presentations, including toxic megacolon, leukemoid reaction, severe hypoalbuminemia, septic shock, and death. ${ }^{26,28-30}$ Several epidemic outbreaks occurred throughout North America during the mid-1900s and mid-2000s, which were attributable to this hypervirulent $C$. diffile strain. ${ }^{31,32}$ According to the Society for Healthcare Epidemiology of America and the Infectious Diseases Society of America, metronidazole remains the initial drug of choice for mildto-moderate CDI, and oral vancomycin for severe CDI. ${ }^{26,28}$ However, recurrence and relapse of CDI, even after repeated cycles of antibiotic therapy, has emerged as a major public health problem. ${ }^{33}$ Fecal microbiota transplantation has been increasingly studied. ${ }^{34-36}$ Cammarota et al ${ }^{34}$ conducted an RCT involving 39 patients with recurrent $\mathrm{CDI}$ (20 patients receiving fecal transplantation and 19 patients receiving vancomycin) and reported significantly higher rates of resolution with the use of fecal transplantation ( $90 \%$ versus $26 \%, P<0.0001)$.

Given the high morbidity and mortality of the CDI, and the rising incidence despite adequate antibiotic therapy, efforts to prevent rather than treat CDI are paramount. Several approaches have been suggested to prevent the transmission of $C$. difficile; however, the mainstay remains early detection and isolation, contact precautions, and appropriate hand hygiene. The use of environmental cleaning disinfectants and chlorhexidine patient baths has also been studied, but has shown only limited success. ${ }^{12,13}$

Probiotics, which are living commensal microorganisms and part of the normal host intestinal flora, has been shown to exert a protective effect on the gastrointestinal tract. The mechanism by which probiotics work has not been fully elucidated, but various mechanisms have been proposed.
Commensal bacteria inhibit enteric pathogens and may help suppress the growth and invasion of pathogenic bacteria, thereby improving intestinal barrier function. ${ }^{37}$ Probiotics also modulate proinflammatory cytokines, which help regulate immune responses and maintain homeostasis. ${ }^{37,38}$ Probiotic supplementation may also allow the acquisition and subsequent population of the gastrointestinal tract with normal commensal bacterial flora, modulating the inflammatory balance, and as a result, decrease the development of CDAD in patients receiving antibiotics..$^{37,38}$

Probiotics have been extensively studied and shown to have a therapeutic role in various gastrointestinal conditions, including diarrhea. Ford et $\mathrm{al}^{39}$ published a meta-analysis, which included 23 RCTs involving 2,575 patients with irritable bowel syndrome, and reported that $21 \%$ of patients experienced improved symptoms with probiotics $(\mathrm{RR}=0.79$; $95 \% \mathrm{CI}, 0.70-0.89 ; P<0.01)$. Shen et $\mathrm{al}^{40}$ published a metaanalysis including 12 RCTs involving 723 patients with inflammatory bowel disorder (649 had ulcerative colitis, 74 had Crohn's disease). Probiotics were effective in inducing remission in patients with ulcerative colitis $(\mathrm{RR}=1.80$; 95\% CI, 1.36-2.39; $P=0.01$ ); however, it was less effective in patients with Crohn's disease ( $\mathrm{RR}=0.89 ; 95 \% \mathrm{CI}$, $0.70-1.13 ; P=0.35) .{ }^{40}$ Salari et al ${ }^{41}$ published a meta-analysis that included 19 RCTs including 3,867 children with acute diarrhea, and reported a significant reduction in the duration of diarrhea (weighted mean difference [WMD] $=-0.67 ; 95 \%$ CI, -0.95 to $-0.38 ; P<0.0001)$ and the duration of fever (WMD $=-0.18 ; 95 \% \mathrm{CI},-0.34$ to $-0.02 ; P=0.0246$ ).

Although CDI is closely linked to the use of broadspectrum antibiotics that disrupt the normal intestinal flora, probiotics as adjunct therapy along with antibiotics has been considered in the prevention of CDI and its complications. The current meta-analysis found that probiotics are associated with a $60.5 \%$ reduction in the incidence of CDAD. ${ }^{17,18}$ The use of Lactobacillus, Saccharomyces, and a mixture of different probiotics all significantly reduced the risk of CDAD $(63.7 \%$, $58.5 \%$, and $58.2 \%$ risk reduction, respectively). There was a $59.5 \%$ risk reduction among adults and a $65.9 \%$ risk reduction among children. A $61 \%$ risk reduction was observed in hospitalized inpatients. In the lone study addressing outpatient treatment with probiotics, a $69.4 \%$ reduction was observed but was not statistically significant. ${ }^{42}$

The efficacy of probiotics has been widely studied in a variety of RCTs; however, the adverse events for probiotic therapy are not well documented. In a comprehensive systematic review conducted by the Agency for Healthcare Research and Quality, a third of the 622 published studies 
provided only nonspecific statements indicating that probiotics were well-tolerated, while most other articles indicated only the presence or absence of one or more specific adverse events, but failed to provide specific details. ${ }^{43}$ Although some case studies have reported fungemia, bacteremia, and sepsis associated with probiotic use, the incidences of these adverse events are inconsistent and not statistically significant across studies. ${ }^{43}$ Most studies included in this meta-analysis provided minimal nonspecific statements about adverse events, although some studies did report no statistical significance between patients receiving probiotics and the control group with respect to nausea, abdominal cramping, constipation, and urticaria. ${ }^{44-47}$ Several studies even noted that probiotics were associated with decrease in length of stay, fever, and nausea/vomiting. ${ }^{44,48}$

Although the results of this meta-analysis are significant, there are limitations to these results, given the variation and heterogeneity of the RCTs analyzed. The enrollment criteria used in each study differed with regard to patient age, comorbidities, and health care setting. The specific strain, dosage, and duration of probiotic also differed between studies, as well as the concurrent antibiotic regimen of the patients. The test used for diagnosing CDI and the follow-up period also varied between studies. Additional studies to determine the optimal dose and particular strain of probiotic as well as the long-term effects of probiotics are required. Most existing studies have included individuals of all age groups, and very few trials have specifically studied elderly patients who are at highest risk of developing CDI and CDAD. Furthermore, almost all studies explicitly stated that immunocompromised patients and patients with a history of major gastrointestinal surgery were excluded from the study, and the remainder were nonspecific as to whether or not these patients were included. Additional randomized placebo-controlled trials more clearly assessing the safety and efficacy of probiotics in this particular population are needed.

\section{Conclusion}

Despite the aforementioned limitations, this study found that probiotic supplementation is a valuable adjunct in the routine care of patients receiving antibiotic therapy. Given the high morbidity and mortality associated with CDI and CDAD, the significant reduction in the incidence of CDAD achieved with probiotic supplementation and the apparent lack of significant negative side effects should prompt physicians to consider these readily available, low-cost supplements as an effective and potentially routine therapy for patients receiving antibiotic therapy.

\section{Disclosure}

The authors report no conflicts of interest in this work.

\section{References}

1. Sun X, Hirota SA. The roles of host and pathogen factors and the innate immune response in the pathogenesis of Clostridium difficile infection. Mol Immunol. 2015;63:193-202.

2. Carroll KC, Bartlett JG. Biology of Clostridium difficile: implications for epidemiology and diagnosis. Annu Rev Microbiol. 2011;65:501-521.

3. Kyne L, Hamel MB, Polavaram R, Kelly CP. Health care costs and mortality associated with nosocomial diarrhea due to Clostridium difficile. Clin Infect Dis. 2002;34:346-353.

4. O'Brien JA, Lahue BJ, Caro JJ, Davidson DM. The emerging infectious challenge of Clostridium difficile-associated disease in Massachusetts hospitals: clinical and economic consequences. Infect Control Hosp Epidemiol. 2007;28:1219-1227.

5. Barbut F, Meynard JL, Guiguet M, et al. Clostridium difficile-associated diarrhea in HIV-infected patients: epidemiology and risk factors. J Acquir Immune Defic Syndr Hum Retrovirol. 1997;16:176-181.

6. Barbut F, Petit JC. Epidemiology of Clostridium difficile-associated infections. Clin Microbiol Infect. 2001;7:405-410.

7. Brown E, Talbot GH, Axelrod P, Provencher M, Hoegg C. Risk factors for Clostridium difficile toxin-associated diarrhea. Infect Control Hosp Epidemiol. 1990;11:283-290.

8. Hutin Y, Molina JM, Casin I, et al. Risk factors for Clostridium difficile-associated diarrhoea in HIV-infected patients. AIDS. 1993;7: 1441-1447.

9. Loo VG, Bourgault AM, Poirier L, et al. Host and pathogen factors for Clostridium difficile infection and colonization. $N$ Engl J Med. 2011;365:1693-1703.

10. Pakyz AL, Jawahar R, Wang Q, Harpe SE. Medication risk factors associated with healthcare-associated Clostridium difficile infection: a multilevel model case-control study among 64 US academic medical centres. J Antimicrob Chemother. 2014;69:1127-1131.

11. Brown KA, Khanafer N, Daneman N, Fisman DN. Meta-analysis of antibiotics and the risk of community-associated Clostridium difficile infection. Antimicrob Agents Chemother. 2013;57:2326-2332.

12. Mayfield JL, Leet T, Miller J, Mundy LM. Environmental control to reduce transmission of Clostridium difficile. Clin Infect Dis. 2000;31: 995-1000.

13. Rupp ME, Cavalieri RJ, Lyden E, et al. Effect of hospital-wide chlorhexidine patient bathing on healthcare-associated infections. Infect Control Hosp Epidemiol. 2012;33:1094-1100.

14. Sullivan A, Nord CE. Probiotics in human infections. J Antimicrob Chemother. 2002;50:625-627.

15. Guarner F, Khan AG, Garisch J, et al. World Gastroenterology Organisation Global Guidelines: probiotics and prebiotics October 2011. J Clin Gastroenterol. 2012;46:468-481.

16. Hempel S, Newberry SJ, Maher AR, et al. Probiotics for the prevention and treatment of antibiotic-associated diarrhea: a systematic review and meta-analysis. JAMA. 2012;307:1959-1969.

17. Johnston BC, Ma SS, Goldenberg JZ, et al. Probiotics for the prevention of Clostridium difficile-associated diarrhea: a systematic review and meta-analysis. Ann Intern Med. 2012;157:878-888.

18. Goldenberg JZ, Ma SS, Saxton JD, et al. Probiotics for the prevention of Clostridium difficile-associated diarrhea in adults and children. Cochrane Database Syst Rev. 2013;5:CD006095.

19. Allen SJ, Wareham K, Wang D, et al. A high-dose preparation of lactobacilli and bifidobacteria in the prevention of antibiotic-associated and Clostridium difficile diarrhoea in older people admitted to hospital: a multicentre, randomised, double-blind, placebo-controlled, parallel arm trial (PLACIDE). Health Technol Assess. 2013;17:1-140.

20. Shan LS, Hou P, Wang ZJ, et al. Prevention and treatment of diarrhoea with Saccharomyces boulardii in children with acute lower respiratory tract infections. Benef Microbes. 2013;4:329-334. 
21. Wong S, Jamous A, O’Driscoll J, et al. A Lactobacillus casei Shirota probiotic drink reduces antibiotic-associated diarrhoea in patients with spinal cord injuries: a randomised controlled trial. Br J Nutr. 2014;111:672-678.

22. Ouwehand AC, DongLian C, Weijian X, et al. Probiotics reduce symptoms of antibiotic use in a hospital setting: a randomized dose response study. Vaccine. 2014;32:458-463.

23. Hall IC, O'Toole E. Intestinal flora in newborn infants with a description of a new pathogenic anaerobe, Bacillus difficilis. Am J Dis Child. 1935;49:390-402.

24. Kelly CP, Pothoulakis C, LaMont JT. Clostridium difficile colitis. N Engl J Med. 1994;330:257-262.

25. Rupnik M, Wilcox MH, Gerding DN. Clostridium difficile infection: new developments in epidemiology and pathogenesis. Nat Rev Microbiol. 2009;7:526-536.

26. To KB, Napolitano LM. Clostridium difficile infection: update on diagnosis, epidemiology, and treatment strategies. Surg Infect (Larchmt). 2014; 15:490-502.

27. Reveles KR, Lee GC, Boyd NK, Frei CR. The rise in Clostridium difficile infection incidence among hospitalized adults in the United States: 2001-2010. Am J Infect Control. 2014;42:1028-1032.

28. Cohen SH, Gerding DN, Johnson S, et al. Clinical practice guidelines for Clostridium difficile infection in adults: 2010 update by the society for healthcare epidemiology of America (SHEA) and the infectious diseases society of America (IDSA). Infect Control Hosp Epidemiol. 2010;31:431-455

29. Warny M, Pepin J, Fang A, et al. Toxin production by an emerging strain of Clostridium difficile associated with outbreaks of severe disease in North America and Europe. Lancet. 2005;366:1079-1084.

30. He M, Miyajima F, Roberts P, et al. Emergence and global spread of epidemic healthcare-associated Clostridium difficile. Nat Genet. 2013;45:109-113.

31. Zilberberg MD, Shorr AF. Preventing Clostridium difficile infection in the intensive care unit. Crit Care Clin. 2013;29:11-18.

32. Merrigan M, Venugopal A, Mallozzi M, et al. Human hypervirulent Clostridium difficile strains exhibit increased sporulation as well as robust toxin production. J Bacteriol. 2010;192:4904-4911.

33. Mathur H, Rea MC, Cotter PD, Ross RP, Hill C. The potential for emerging therapeutic options for Clostridium difficile infection. Gut Microbes. 2014;5:696-710.

34. Cammarota G, Masucci L, Ianiro G, et al. Randomised clinical trial: faecal microbiota transplantation by colonoscopy vs vancomycin for the treatment of recurrent Clostridium difficile infection. Aliment Pharmacol Ther. 2015;41:835-843.

35. Borody T, Fischer M, Mitchell S, Campbell J. Fecal microbiota transplantation in gastrointestinal disease: 2015 update and the road ahead. Expert Rev Gastroenterol Hepatol. 2015;9(11):1379-1391.

36. Youngster I, Sauk J, Pindar C, et al. Fecal microbiota transplant for relapsing Clostridium difficile infection using a frozen inoculum from unrelated donors: a randomized, open-label, controlled pilot study. Clin Infect Dis. 2014;58:1515-1522.

37. Rohde CL, Bartolini V, Jones N. The use of probiotics in the prevention and treatment of antibiotic-associated diarrhea with special interest in Clostridium difficile-associated diarrhea. Nutr Clin Pract. 2009;24:33-40.

38. Jones SE, Versalovic J. Probiotic Lactobacillus reuteri biofilms produce antimicrobial and anti-inflammatory factors. BMC Microbiol. 2009;9:35.

39. Ford AC, Quigley EM, Lacy BE, et al. Efficacy of prebiotics, probiotics, and synbiotics in irritable bowel syndrome and chronic idiopathic constipation: systematic review and meta-analysis. Am J Gastroenterol. 2014;109:1547-1561.

40. Shen J, Zuo ZX, Mao AP. Effect of probiotics on inducing remission and maintaining therapy in ulcerative colitis, Crohn's disease, and pouchitis: meta-analysis of randomized controlled trials. Inflamm Bowel Dis. 2014;20:21-35.
41. Salari P, Nikfar S, Abdollahi M. A meta-analysis and systematic review on the effect of probiotics in acute diarrhea. Inflamm Allergy Drug Targets. 2012;11:3-14.

42. Duman DG, Bor S, Ozutemiz O, et al. Efficacy and safety of Saccharomyces boulardii in prevention of antibiotic-associated diarrhoea due to Helicobacterpylori eradication. Eur J Gastroenterol Hepatol. 2005; 17:1357-1361.

43. Hempel S, Newberry S, Ruelaz A, et al. Safety of probiotics used to reduce risk and prevent or treat disease. Evid Rep Technol Assess (Full Rep). 2011;1-645.

44. Thomas MR, Litin SC, Osmon DR, Corr AP, Weaver AL, Lohse CM. Lack of effect of Lactobacillus GG on antibiotic-associated diarrhea: a randomized, placebo-controlled trial. Mayo Clin Proc. 2001;76: 883-889.

45. Beausoleil M, Fortier N, Guenette S, et al. Effect of a fermented milk combining Lactobacillus acidophilus C11285 and Lactobacillus casei in the prevention of antibiotic-associated diarrhea: a randomized, double-blind, placebo-controlled trial. Can J Gastroenterol. 2007;21:732-736.

46. Cindoruk M, Erkan G, Karakan T, Dursun A, Unal S. Efficacy and safety of Saccharomyces boulardii in the 14-day triple anti-Helicobacter pylori therapy: a prospective randomized placebo-controlled double-blind study. Helicobacter. 2007;12:309-316.

47. Lonnermark E, Friman V, Lappas G, Sandberg T, Berggren A, Adlerberth I. Intake of Lactobacillus plantarum reduces certain gastrointestinal symptoms during treatment with antibiotics. J Clin Gastroenterol. 2010;44:106-112.

48. McFarland LV, Surawicz CM, Greenberg RN, et al. Prevention of betalactam-associated diarrhea by Saccharomyces boulardii compared with placebo. Am J Gastroenterol. 1995;90:439-448.

49. Surawicz CM, Elmer GW, Speelman P, McFarland LV, Chinn J, van BG. Prevention of antibiotic-associated diarrhea by Saccharomyces boulardii: a prospective study. Gastroenterology. 1989;96:981-988.

50. Arvola T, Laiho K, Torkkeli S, et al. Prophylactic Lactobacillus GG reduces antibiotic-associated diarrhea in children with respiratory infections: a randomized study. Pediatrics. 1999;104:e64.

51. Plummer S, Weaver MA, Harris JC, Dee P, Hunter J. Clostridium difficile pilot study: effects of probiotic supplementation on the incidence of C. difficile diarrhoea. Int Microbiol. 2004;7:59-62.

52. Kotowska M, Albrecht P, Szajewska H. Saccharomyces boulardii in the prevention of antibiotic-associated diarrhoea in children: a randomized double-blind placebo-controlled trial. Aliment Pharmacol Ther. 2005;21:583-590.

53. Can M, Besirbellioglu BA, Avci IY, Beker CM, Pahsa A. Prophylactic Saccharomyces boulardii in the prevention of antibiotic-associated diarrhea: a prospective study. Med Sci Monit. 2006;12:I19-I22.

54. Hickson M, D’Souza AL, Muthu N, et al. Use of probiotic Lactobacillus preparation to prevent diarrhoea associated with antibiotics: randomised double blind placebo controlled trial. BMJ. 2007;335:80.

55. Rafiq R, Pandey D, Osman SM, Masood R, Donepudi I, Norkus E. Prevention of Clostridium difficile ( $C$-difficile) diarrhea with probiotic in hospitalized patients treated with antibiotics [abstract]. Gastroenterology. 2007;132(Suppl 2):A187.

56. Miller M, Florencio S, Eastmond J, Reynolds S. Results of 2 prospective randomized studies of Lactobacillus GG to prevent $C$. difficile infection in hospitalized adults receiving antibiotics [abstract]. Abstract of the Interscience Conference on Antimicrobial Agents and Chemotherapy. 2008;48:578-579.

57. Ruszczynski M, Radzikowski A, Szajewska H. Clinical trial: effectiveness of Lactobacillus rhamnosus (strains E/N, Oxy and Pen) in the prevention of antibiotic-associated diarrhoea in children. Aliment Pharmacol Ther. 2008;28:154-161.

58. Safdar N, Barigala R, Said A, McKinley L. Feasibility and tolerability of probiotics for prevention of antibiotic-associated diarrhoea in hospitalized US military veterans. J Clin Pharm Ther. 2008;33: 663-668. 
59. Wenus C, Goll R, Loken EB, Biong AS, Halvorsen DS, Florholmen J. Prevention of antibiotic-associated diarrhoea by a fermented probiotic milk drink. Eur J Clin Nutr. 2008;62:299-301.

60. Gao XW, Mubasher M, Fang CY, Reifer C, Miller LE. Dose-response efficacy of a proprietary probiotic formula of Lactobacillus acidophilus CL1285 and Lactobacillus casei LBC80R for antibiotic-associated diarrhea and Clostridium difficile-associated diarrhea prophylaxis in adult patients. Am J Gastroenterol. 2010;105:1636-1641.

61. Sampalis J, Psaradellis E, Rampakakis E. Efficacy of BIO K+ CL1285 in the reduction of antibiotic-associated diarrhea - a placebo controlled double-blind randomized, multi-center study. Arch Med Sci. 2010;6:56-64
62. Pozzoni P, Riva A, Bellatorre AG, et al. Saccharomyces boulardii for the prevention of antibiotic-associated diarrhea in adult hospitalized patients: a single-center, randomized, double-blind, placebo-controlled trial. Am J Gastroenterol. 2012;107:922-931.

63. Selinger CP, Bell A, Cairns A, Lockett M, Sebastian S, Haslam N. Probiotic VSL\#3 prevents antibiotic-associated diarrhoea in a doubleblind, randomized, placebo-controlled clinical trial. J Hosp Infect. 2013;84:159-165.

International Journal of General Medicine

\section{Publish your work in this journal}

The International Journal of General Medicine is an international, peer-reviewed open-access journal that focuses on general and internal medicine, pathogenesis, epidemiology, diagnosis, monitoring and treatment protocols. The journal is characterized by the rapid reporting of reviews, original research and clinical studies across all disease areas.

\section{Dovepress}

A key focus is the elucidation of disease processes and management protocols resulting in improved outcomes for the patient. The manuscript management system is completely online and includes a very quick and fair peer-review system. Visit http://www.dovepress.com/ testimonials.php to read real quotes from published authors.

Submit your manuscript here: http://www.dovepress.com/international-journal-of-general-medicine-journal 